\title{
FINITELY GENERATED RIGHT IDEALS OF TRANSFORMATION NEAR-RINGS
}

\author{
S. D. SCOTT
}

\begin{abstract}
If $V$ is an additive group, $M_{0}(V)$ the near-ring of zero-fixing maps of $V$ into $V$, then the finitely generated right ideals of $M_{0}(V)$ are easily characterised. These are just the annihilators of subsets of $V$. Moreover, finitely generated right ideals of $M_{0}(V)$ are generated by a single element.
\end{abstract}

Throughout this paper all groups will be written additively but this does not imply commutativity. All near-rings considered will be zero-symmetric and left distributive. The near-ring of all zero-fixing maps of a group $V$ into itself under composition and pointwise addition will be denoted by $M_{0}(V)$.

Let $N$ be a near-ring, and $S$ a nonempty subset of $N$. The right ideal of $N$ generated by $S$ will be denoted by $R(S)$. If $H$ is a right ideal of $N$ and $H=R(S)$ where $S$ is a finite nonempty subset of $N$, then the right ideal $H$ will be said to be finitely generated. In the case where $S$ consists of the single element $\gamma$ of $N, R(S)$ will be denoted by $R(\gamma)$. Clearly if $S=\left\{\gamma_{1}, \ldots, \gamma_{k}\right\}$ where $k>1$ is an integer, then $R(S)=R\left(\gamma_{1}\right)+R\left(\gamma_{2}\right)+\cdots+R\left(\gamma_{k}\right)$. The following theorem will be proved.

THEOREM 1. Let $V$ be a group and $H$ a right ideal of $M_{0}(V)$. The right ideal $H$ is finitely generated if, and only if, $H=(0: L)$ where $L$ is some nonempty subset of $V$. Furthermore if $H$ is finitely generated then there exists $\gamma$ in $H$ such that $H=R(\gamma)$.

This theorem will be proved with the aid of certain propositions and lemmas.

Let $V$ be a group. Suppose $S_{1}$ and $S_{2}$ are subsets of $V$ such that $S_{1} \cup S_{2}=V$ and $S_{1} \cap S_{2}=\{0\}$ then, as every function $\alpha$ of $V$ into $V$ that fixes zero can be expressed as a sum $\alpha_{1}+\alpha_{2}$, where $\alpha_{i}, i=1,2$, are functions of $V$ into $V$ fixing zero and $\alpha_{1}$ is zero on $S_{1}$ and $\alpha_{2}$ is zero on $S_{2}$, the following proposition holds.

Proposition 2. Let $V$ be a group and $S_{1}$ and $S_{2}$ subsets of $V$ such that $S_{1} \cup S_{2}=V$, and $S_{1} \cap S_{2}=\{0\}$. It follows that $M_{0}(V)=\left(0: S_{1}\right) \oplus\left(0: S_{2}\right)$.

Suppose $L$ is a nonempty subset of a group $V$. Let $L_{1}=L \cup\{0\}$, and $L_{2}=$ $(V \backslash L) \cup\{0\}$. Clearly the subsets $L_{1}$ and $L_{2}$ of $V$ satisfy the conditions of the above proposition and therefore, $\left(0: L_{1}\right) \oplus\left(0: L_{2}\right)=M_{0}(V)$. The identity 1 of $M_{0}(V)$ can therefore be written as $e_{1}+e_{2}$ where $e_{1}$ is in $\left(0: L_{1}\right)$, and $e_{2}$ is in $\left(0: L_{2}\right)$. Also if $\alpha$ is in $M_{0}(V)$, then $e_{1} \alpha+e_{2} \alpha=\alpha$. Thus $e_{1} M_{0}(V)=\left(0: L_{1}\right)$, and $\left(0: L_{1}\right)(=(0: L))$ is generated by the single element $e_{1}$ (if $L=\{0\}$, or $V$, then

Received by the editors May 31, 1979.

AMS (MOS) subject classifications (1970). Primary 16A76; Secondary 16A66, 16A42. 
$(0: L)$ is $M_{0}(V)$, or $\{0\}$, and the result holds with $e_{1}=1$, or 0$)$. It now follows that right ideals of $M_{0}(V)$ of the form $(0: L)$ ( $L$ a nonempty subset of $V$ ) are finitely generated and the 'if' part of Theorem 1 is established.

If $\gamma$ is an element of $M_{0}(V)$ then we denote the set $\{v \in V: v \gamma=0\}$ by $Z(\gamma)$ (see [2, p. 200]).

LEMMA 3. If $V$ is a group and $\gamma$ an element of $M_{0}(V)$ then $R(\gamma)=(0: Z(\gamma))$.

Proof. Let $\Lambda=(V \backslash Z(\gamma)) \cup\{0\}$. By Proposition 2, $M_{0}(V)=(0: Z(\gamma)) \oplus$ $(0: \Lambda)$. Clearly $\gamma$ is in $(0: Z(\gamma))$ and, $R(\gamma) \leqslant(0: Z(\gamma))$. Now $1=e_{1}+e_{2}$, where $e_{1}$ is in $(0: Z(\gamma))$, and $e_{2}$ is in $(0: \Lambda)$. Also, since, $e_{2} M_{0}(V)=(0: \Lambda), R\left(e_{2}\right)=$ $(0: \Lambda)$. Consider the element $\gamma+e_{2}$ of $M_{0}(V)$. If $v$ is in $(V \backslash Z(\gamma)) \cup\{0\}$, then $v e_{2}=0$ and $v \gamma \neq 0$, unless $v=0$. If $v$ is in $Z(\gamma)$, then since $Z\left(e_{2}\right)=(V \backslash Z(\gamma)) \cup$ $\{0\}$, it follows that $v e_{2} \neq 0$, unless $v=0$. Thus if $v$ in $V$ is such that $v\left(\gamma+e_{2}\right)=0$, then it must follow that $v=0$. Now $R\left(\gamma+e_{2}\right)=M_{0}(V)$, or $R\left(\gamma+e_{2}\right)$ is contained in a maximal right ideal of $M_{0}(V)$. If $R\left(\gamma+e_{2}\right)$ is contained in a maximal right ideal of $M_{0}(V)$ by a result of M. Johnson [1] (see also Pilz [2, p. 200]) it follows that there exists $v \neq 0$ in $V$ such that $v\left(\gamma+e_{2}\right)=0$. Thus $R\left(\gamma+e_{2}\right)=M_{0}(V)$. Now $R\left(\gamma+e_{2}\right) \leqslant R(\gamma)+R\left(e_{2}\right)$, and since $R(\gamma) \leqslant(0: Z(\gamma))$ and $R\left(e_{2}\right)=(0: \Lambda)$, we conclude that $R(\gamma) \oplus(0: \Lambda)=M_{0}(V)$. Clearly this can only happen if $R(\gamma)=$ $(0: Z(\gamma))$. The lemma is therefore proved.

COROLlaRY. If $\lambda_{1}$ and $\lambda_{2}$ are elements of $M_{0}(V)$ such that $Z\left(\lambda_{1}\right)<Z\left(\lambda_{2}\right)$, then $R\left(\lambda_{2}\right)<R\left(\lambda_{1}\right)$.

By the above lemma, if the last statement of the theorem is proved the rest will follow.

LEMMA 4. Let $V$ be a group and $H$ a finitely generated right ideal of $M_{0}(V)$. There exists $\gamma$ in $H$ such that $R(\gamma)=H$.

Proof. Suppose $P$ is a right ideal of $M_{0}(V)$ generated by a two element subset $\left\{\gamma_{1}, \gamma_{2}\right\}$ of $M_{0}(V)$. If it is established that $P=R\left(\gamma^{\prime}\right)$ for some $\gamma^{\prime}$ in $M_{0}(V)$, then the lemma will follow. Let $\Lambda=\left(V \backslash Z\left(\gamma_{1}\right)\right) \cup\{0\}$. We have, by Proposition 2, that $M_{0}(V)=\left(0: Z\left(\gamma_{1}\right)\right) \oplus(0: \Lambda)$, and by Lemma 3, $R\left(\gamma_{1}\right)=\left(0: Z\left(\gamma_{1}\right)\right)$. Now $\gamma_{2}=\alpha$ $+\beta$ where $\alpha$ is in $\left(0: Z\left(\gamma_{1}\right)\right)$, and $\beta$ is in $(0: \Lambda)$. Take $\gamma^{\prime}=\gamma_{1}+\beta$. Now every $v$ in $V$ is such that either $v \gamma_{1}=0$ or $v \beta=0$. Thus if $v \gamma^{\prime}=0$, it follows that $v \gamma_{1}+v \beta=0$, and $v \gamma_{1}=0$. Hence $Z\left(\gamma^{\prime}\right) \subseteq Z\left(\gamma_{1}\right)$, and by the Corollary of Lemma 3, we conclude that $R\left(\gamma_{1}\right) \leqslant R\left(\gamma^{\prime}\right)$. Thus $-\gamma_{1}+\gamma^{\prime}=\beta$ is in $R\left(\gamma^{\prime}\right)$, and since $\alpha$ is in $\left(0: Z\left(\gamma_{1}\right)\right)=R\left(\gamma_{1}\right)$, we see that $\alpha+\beta=\gamma_{2}$ is in $R\left(\gamma^{\prime}\right)$. Hence $R\left(\gamma^{\prime}\right)>R\left(\gamma_{1}\right)+$ $R\left(\gamma_{2}\right) \geqslant P$. But $R\left(\gamma^{\prime}\right) \leqslant R\left(\gamma_{1}\right)+R(\beta)$ and, since $\alpha$ is in $R\left(\gamma_{1}\right), R\left(\gamma^{\prime}\right)<R\left(\gamma_{1}\right)+$ $R\left(\gamma_{2}\right)(=P)$. Thus $R\left(\gamma^{\prime}\right)=P$ and the lemma follows.

\section{REFERENCES}

1. M. Johnson, Maximal right ideals of transformation near-rings, J. Austral. Math. Soc. 19 (1975), 410-417.

2. G. Pilz, Near-rings, North-Holland, Amsterdam, 1977.

Department of Mathematics, University of Auckland, Auckiand, New Zealand 